\title{
rs3918242 MMP9 gene polymorphism is associated with myocardial infarction in Mexican patients
} T.X. Zagal-Jiménez ${ }^{1}$, R. Ortíz-Alarcón ${ }^{1}$, B. Valente-Acosta ${ }^{1}$, C. Tovilla-Zárate ${ }^{3}$, C. Nostroza-Hernández ${ }^{1}$, O. Pérez-Méndez ${ }^{1}$ and N. Pérez-Hernández ${ }^{1,4}$

1Department of Molecular Biology, Instituto Nacional de Cardiología Ignacio Chávez, Mexico City, Mexico

2Department of Endocrinology, Instituto Nacional de Cardiología Ignacio Chávez, Mexico City, Mexico

3Universidad Juárez Autónoma de Tabasco,

División Académica Multidisciplinaria de Comacalco, Tabasco, Mexico

${ }^{4}$ Graduate School of Genomic Sciences,

Universidad Autónoma de la Ciudad de México, Mexico City, Mexico

*These authors contributed equally to this study.

Corresponding author: N. Pérez-Hernández

E-mail: unicanona@yahoo.com.mx

Genet. Mol. Res. 15 (1): gmr.15017776

Received October 5, 2015

Accepted December 18, 2015

Published March 4, 2016

DOI http://dx.doi.org/10.4238/gmr.15017776

\begin{abstract}
Several studies have demonstrated that matrix metalloproteinases (MMPs) play a major role in atherosclerotic plaque disruption and lead to myocardial infarction (MI). We investigated the association between the MMP1 -1607 1G/2G (rs1799750), MMP3 -1612 5A/6A (rs3025058), and MMP9 -1562 C/T (rs3918242) polymorphisms and the risk of developing $\mathrm{MI}$ in a Mexican mestizo cohort. The genotype analysis was performed using the restriction fragment length polymorphism-polymerase chain reaction technique in a group of 236 patients with a history of $\mathrm{Ml}$ and 285 healthy controls. Similar distributions of rs1799750 and rs3025058 were observed in both groups; however,
\end{abstract}


the MMP9 rs3918242 $T$ allele and the $C T$ genotype were associated with the risk of developing $\mathrm{MI}(\mathrm{OR}=2.32, \mathrm{pC}=0.02$ and $\mathrm{OR}=2.40, \mathrm{pC}=$ 0.02 , respectively). Multiple logistic analysis was performed between $\mathrm{MI}$ patients and controls to estimate the risk, and after adjusting for identified risk factors, the CT + TT genotypes of MMP9 rs3918242 were found to be significantly associated with increased risk of developing $\mathrm{Ml}$ than those with the $C C$ genotype $(O R=2.88, P<0.01)$. In summary, our results reveal that the rs3918242 polymorphism of the MMP9 gene plays a major role in the risk of developing MI.

Key words: Matrix metalloproteinase; Polymorphisms; Myocardial infarction; Atherothrombosis

\section{INTRODUCTION}

Globally, coronary artery disease (CAD) is one of the main causes of mortality, and myocardial infarction (MI) is one of the main clinical presentations of its pathology. $\mathrm{Ml}$ is a complex disease involving multiple genetic and environmental factors. The main environmental factors underlying atherosclerosis and CAD development include smoking, diabetes, hypertension, obesity, and hypercholesterolemia. Recently, numerous studies have provided evidence that genetic polymorphisms play a pivotal role in the development of CAD and MI (Wang et al., 2015).

Current evidence indicates that disturbances in arterial extracellular matrix remodeling play an important role in the atherosclerotic process. The activity of metalloproteinases (MMPs) may contribute to the pathophysiology of atherosclerosis by facilitating the migration of vascular smooth muscle cells into the internal elastic lamina at the intima of the vessel wall, where they proliferate and contribute to plaque establishment and growth (Herzog et al., 1998; Newby, 2005). The MMPs are a family of zinc-dependent endopeptidases responsible for degrading extracellular components (Creemers et al., 2001; Falk, 2006). Each MMP has its own substrate specificity (Ratnikov et al., 2014). Previous studies have suggested that MMPs may play a role in the development of several diseases, such as spontaneous early pregnancy failure (Nissi et al., 2013), solid and hematological malignancies (Chaudhary et al., 2013), and abdominal aortic aneurysms (Duellman et al., 2012). Several polymorphisms in the genes encoding MMP molecules have been studied in cardiovascular diseases (Chehaibi et al., 2014; Olsen et al., 2014; Opstad et al., 2014). It is essential to note that association study results may differ among populations owing to genetic variability between populations, including differences in allele frequencies and linkage disequilibrium structure (Neale and Sham, 2004). The variation in CAD risk between individuals is heritable, indicating that genetic risk factors play a critical role in pathogenesis.

The Mexican population is highly heterogenic. Nevertheless, $98 \%$ of the population are Mestizos, the descendants of the native Amerindians and immigrants from Europe and Africa who arrived in Mexican territory more than 500 years ago, during the period when Mexico was a Spanish colony. The remaining $2 \%$ of the Mexican population comprises other groups, mainly Amerindians (Camacho-Mejorado et al., 2015).

In the current study, we included the coronary calcium scores of the study population in an attempt to exclude controls with subclinical atherosclerosis. This factor has been the main strength of other reports. Therefore, the aim of the present study was to be the first study to determine if there is a significant association between MMP1, MMP3, and MMP9 gene polymorphisms and 
MI in the Mexican Mestizo population. We also investigated the relationship between MMP gene polymorphisms and cardiovascular risk factors.

\section{MATERIAL AND METHODS}

\section{Subjects}

A letter of consent was obtained from all participants. The study complies with the Declaration of Helsinki and was approved by the Ethics and Research Committee of Instituto Nacional de Cardiología "Ignacio Chávez". This study included 236 Mexican Mestizo patients with a history of MI (194 men and 42 women). MI was clinically characterized by classic angina symptoms and defined as an elevation of $1 \mathrm{~mm}$ or more of the ST-segment, as well as the development of a $\mathrm{Q}$ wave in two or more contiguous electrocardiographic records, and/or thrice the upper limit of serum creatine phosphokinase MB isoenzyme (normal value $=0.6-6.3 \mathrm{ng} / \mathrm{mL}$ ) in at least one blood sample.

A group of 285 healthy unrelated individuals ( 134 men and 151 women) who did not have any history compatible with stable angina or a previous diagnosis of systemic or cardiovascular disease (corroborated clinically and by electrocardiography) was used as a control group. The coronary calcium score was determined by multidetector computed tomography to identify subclinical atherosclerosis; all control individuals were required to have a value of zero. Anthropometric measures were recorded and medical history information was collected on traditional risk factors in all included participants. We used clinical criteria to identify type 2 diabetes mellitus patients: a serum glucose level of $126 \mathrm{mg} / \mathrm{dL}$ or higher in two samples, a previous diagnosis, or current hypoglycemic treatment and/or insulin. Hypertensive patients were identified by a previous diagnosis or by participation in active antihypertensive therapy. Dyslipidemia was defined as total cholesterol of $200 \mathrm{mg} / \mathrm{dL}$ or higher, low-density lipoprotein cholesterol $\geq 130 \mathrm{mg} / \mathrm{dL}$, or serum triglycerides of 150 $\mathrm{mg} / \mathrm{dL}$ or higher. Active smokers were identified by a consumption habit of five or more tobacco cigarettes per day, or abstinence for less than twelve months.

Individuals were considered to be active alcohol drinkers if they drank more than $6 \mathrm{~g}$ daily. Body mass index was calculated by a standard formula (weight in $\mathrm{kg} / \mathrm{squared}$ height). Coronary angiography was performed in all patients. The extent of CAD was estimated by determination of the number of stenotic coronary arteries, classified as one-, two-, or three-vessel disease. Involvement of two vessels meant significant stenosis in two coronary vessels or a narrowing of $\geq 50 \%$ in the left main trunk coronary artery, and involvement of three vessels meant significant stenosis in three coronary vessels. Involvement of one vessel was considered hemodynamically significant with more than a $50 \%$ reduction in the diameter of an important vessel (right coronary, left anterior descending, or circumflex).

For this study, a Mexican Mestizo was defined as a person born in Mexico who was the descendant of the original autochthonous inhabitants of the region and of individuals, mainly Spaniards, of Caucasian and/or African origin, who came to America during the colonization period. Therefore, all subjects included were ethnically matched, and were considered to be Mexican Mestizo only if they were at least third-generation Mexicans.

\section{DNA extraction}

The Lahiri and Nurnberger method was used to isolate genomic DNA from blood cells (Lahiri and Nurnberger, 1991). 


\section{Polymorphism analysis (MMP1, MMP3, and MMP9)}

MMP1 -1607 1G/2G (rs1799750), MMP3 -1612 5A/6A (rs3025058), and MMP9 -1562 C/T (rs3918242) polymorphisms were determined using the restriction fragment length polymorphismpolymerase chain reaction (RFLP-PCR) technique (Table 1).

\section{Statistical analysis}

The allele and genotype frequencies of the polymorphisms were obtained by direct counting. The Hardy-Weinberg equilibrium (HWE) was calculated using the chi-square test. Statistical analysis was carried out with SPSS for Windows. Comparison of numerical variables between the groups was performed using the Mann-Whitney U-test; data are reported as medians and 25 and 75 percentiles. Categorical variables were analyzed using the chi-squared or Fisher test as required, and are reported as absolute frequencies and proportions. Statistical significance was set at $\mathrm{P}<0.05$. Multiple logistic analysis was used to test for association of polymorphisms with $\mathrm{MI}$ adjusted for gender, smoking habits, diabetes mellitus, and hypertension. All P values quoted were corrected $(\mathrm{pC})$ by the Bonferroni test for multiple comparisons.

\section{RESULTS}

\section{Sample characteristics}

The demographic and clinical characteristics of the study groups are presented in Table 2. As expected, type 2 diabetes mellitus, hypertension, and smoking habits were more frequent in $\mathrm{MI}$ patients than in healthy controls. In the patient group, $6.3 \%$ showed no occluded vessels, $32.6 \%$ exhibited a one-vessel occlusion, $30.1 \%$ a two-vessel occlusion, and $30.9 \%$ a three-vessel occlusion. Moreover, $9.7 \%$ of patients presented with coronary calcification.

\section{Allele and genotype frequencies}

The allele and genotype frequencies of MMP1 -1607 1G/2G (rs1799750), MMP3 -1612 $5 A / 6 A$ (rs3025058), and MMP9 -1562 C/T (rs3918242) gene polymorphisms in healthy controls and $\mathrm{MI}$ patients are shown in Table 3. The observed and expected frequencies of all polymorphic sites were in HWE. The distribution of MMP1 -1607 2G/1G (rs1799750) and MMP3 -1612 5A/6A (rs3025058) was similar in both groups; however, there was a difference in the MMP9 $-1562 \mathrm{C} / T$ (rs3918242) gene polymorphism distribution between the MI patients and the healthy controls. Patients showed increased frequencies of the $T$ allele and the CT genotype compared with the healthy controls $(\mathrm{OR}=2.32 ; 95 \% \mathrm{Cl}=1.15-4.73 ; \mathrm{pC}=0.02$ and $\mathrm{OR}=2.40 ; 95 \% \mathrm{Cl}=1.17-4.97 ; \mathrm{pC}=0.02$, respectively). However, the MMP9 -1562 C/T (rs3918242) $C$ allele and CC genotype frequencies decreased in $\mathrm{MI}$ patients compared with the healthy controls $(\mathrm{OR}=0.43 ; 95 \% \mathrm{Cl}=0.21-0.87$; $\mathrm{pC}$ $=0.02$ and $\mathrm{OR}=0.42 ; 95 \% \mathrm{Cl}=0.20-0.86 ; \mathrm{pC}=0.02$, respectively). Multiple logistic analysis was used to estimate the risk between the $\mathrm{Ml}$ patients and the controls adjusted by gender, smoking habits, diabetes mellitus, and hypertension. In this analysis, the MMP9 -1562 (rs3918242) CT + TT genotypes were significantly associated with the risk of developing $\mathrm{MI}$ compared with the CC genotype $(\mathrm{OR}=2.88 ; 95 \% \mathrm{Cl}=1.18-6.99 ; \mathrm{P}<0.01)$ (data not shown). 


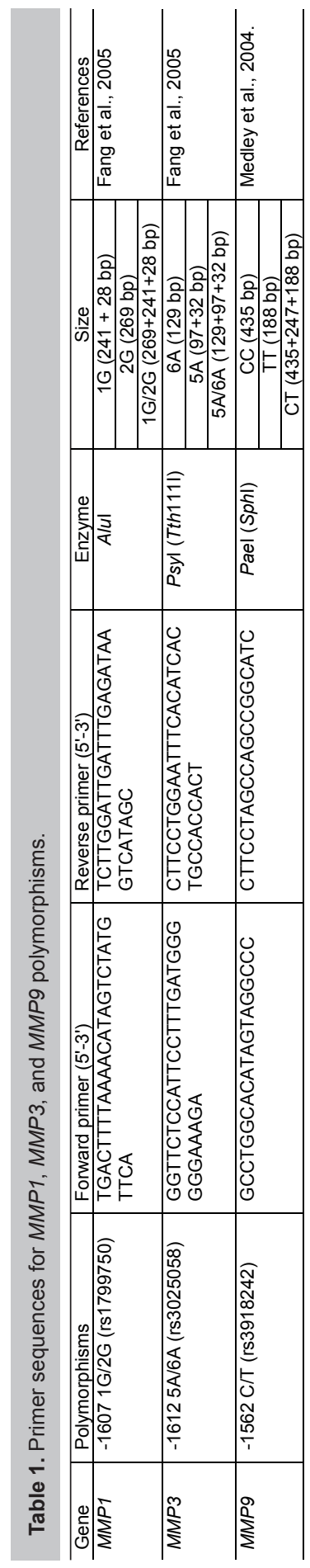


J.M. Rodríguez-Pérez et al.

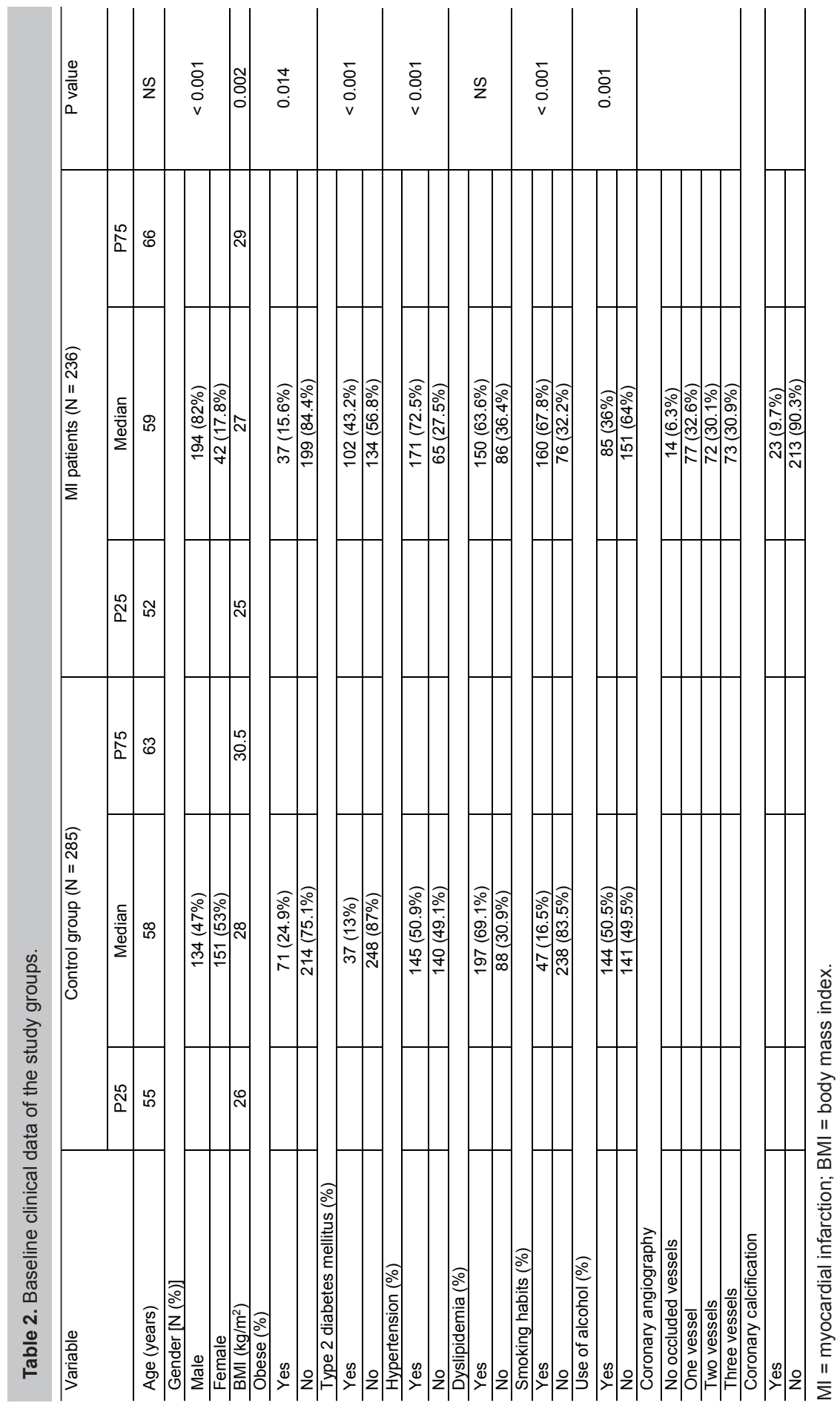




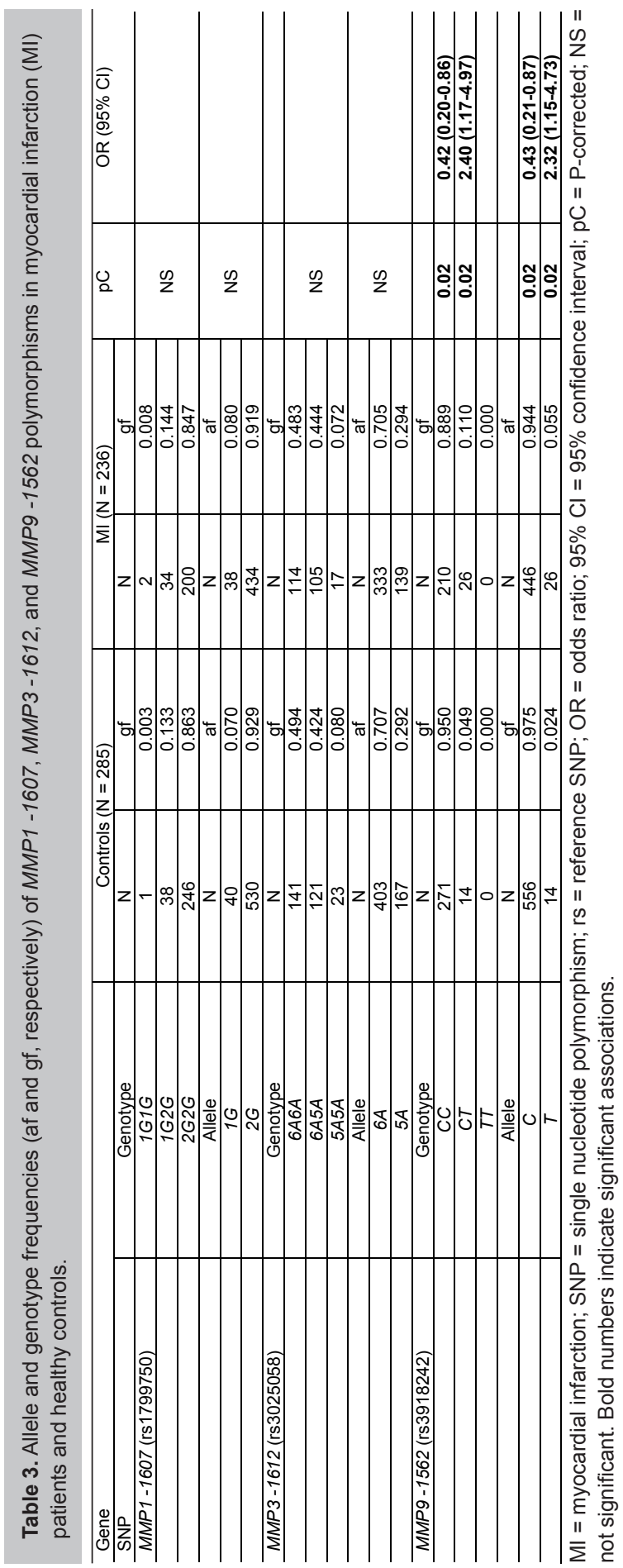




\section{DISCUSSION}

The pathophysiology of $\mathrm{MI}$ includes the transition of a stable coronary atherosclerotic lesion onto a ruptured plaque. After the rupture, a thrombus is formed, which is conducive to a hypoxic lesion and is clinically manifested as MI. MMPs, mainly synthesized by the infiltrating cells, degrade the extracellular matrix and contribute to the weakening of the fibrous cap and its subsequent rupture (Brown et al., 1995). It is noteworthy that polymorphisms in MMP genes have been studied in several diseases with contradictory results (Alp et al., 2009; Zhi et al., 2010; Alp et al., 2011). Association of these polymorphisms has been observed in CAD (Opstad et al., 2013), and intracerebral hemorrhage (Yang et al., 2014). In the present study, we analyzed three MMP gene polymorphisms in Mexican Mestizo patients with MI. The distribution of the MMP1 -1607 1G/2G (rs1799750) and MMP3 -1612 5A/6A (rs3025058) polymorphisms was similar in both groups. However, we found a different distribution of the -1562 (rs3918242) polymorphism of the MMP9 gene compared with the healthy controls. The increased frequencies of the $T$ allele and CT genotype of this polymorphism supports its role as a genetic susceptibility marker for MI in the studied population. In fact, increased levels of MMP9 in the atherosclerotic plaques of coronary arteries have been reported (Pasterkamp et al., 2000).

The polymorphic sites were selected based on previous association studies in cardiovascular diseases and their important role at the functional level. The MMP1 $1 G$ and $2 G$ alleles differ in the insertion of one guanine nucleotide. The polymorphism site is located at position -1607 relative to the transcriptional start site of the gene. The transcriptional activity of the $2 G$ allele is 20 times stronger than that of $1 \mathrm{G}$. Hence, carriers of the former allele will overexpress MMP1, which can lead to a diminished collection of collagen. This is known to induce atherosclerosis (Ye, 2000).

The $5 A$ and $6 A$ alleles of the MMP3 gene differ in a single adenine insertion and include either five or six adenine nucleotides at position -1612 of the promoter region. Both homozygous and heterozygous carriers of the $5 A$ allele have a higher risk of CAD than $6 A$ carriers, since the transcriptional activity of the $5 A$ allele is twice as active. (Liu et al., 2003; Liu et al., 2005).

MMP9 polymorphisms are caused by a single C/T base change at -1562 (the promoter region). Unlike the $C$ allele, the $T$ allele is associated with $C A D$, since it enhances gene expression (Ye, 2000).

Román-García et al. (2009) evaluated three MMP1 polymorphisms (rs11292517, rs1144393, and rs514921) in 261 male patients with MI. They found no association between MI and the MMP1 polymorphisms. These results are in agreement with our study, since we also found that the same gene (albeit a different variant) was not associated with the disease.

In another report, Sakowicz et al. (2013) analyzed 17 polymorphisms in 15 candidate genes. Among them, MMP3 -1612 5A/6A (rs3025058) and MMP9 -1562 (rs3918242) gene polymorphisms were examined in a cohort of 271 patients with $\mathrm{Ml}$ and 141 healthy controls in a white Caucasian population. They reported that the MMP3 -1612 5A/6A (rs3025058) polymorphism was associated with advanced stenosis in arterial vessels, whereas the MMP9 -1562 (rs3918242) T allele was associated with hypertension and a higher risk of MI. In our study, we did not detect an association between the -1612 5A/6A (rs3025058) polymorphism of the MMP3 gene and the disease. Sakowicz et al. (2013)'s findings on the MMP9 -1562 (rs3918242) polymorphism were in line with our results; when a logistic regression analysis was performed to determine the association between cardiovascular risk factors and the CT + TT genotypes of this polymorphic site, we found an association only with MMP9 -1562 (rs3918242).

In addition, Qintao et al. (2014) reported a case-control study in which they examined 
nine polymorphisms of the MMP1 gene in 438 healthy controls and 411 patients with CAD from a Chinese Han population. Their analysis revealed a weak association between the rs 1799750 polymorphism and CAD, while we found no such association with the same polymorphism. This discrepancy may be related to differences in genetic background in the study population or in the selection of patients and healthy controls. Furthermore, Sewelam et al. (2013) studied the association between the MMP3 and MMP9 gene polymorphisms and MI in an Egyptian population and reported an association of the MMP3 -1612 5A/6A (rs3025058) and MMP9 -1562 (rs3918242) $C T+T T$ genotypes and the risk of developing MI. In our study, the same polymorphic sites were analyzed. However, we only detected an association in the CT + TT genotypes of the MMP9 -1562 (rs3918242) polymorphism. The principal differences between these studies were the number of patients included and the number of polymorphisms analyzed. In their report, Sewelam et al. (2013) included only 40 patients compared with the 236 patients analyzed in our study. We examined three polymorphisms in different MMP genes, whereas Sewelam et al. (2013) analyzed only two polymorphisms. The association between MMP1 -1607 1G/2G (rs1799750) and MMP3 -1612 5A/6A (rs3025058) polymorphisms and MI was not corroborated in a large Caucasian population study, and only the MMP9 -1562 (rs3918242) polymorphism was associated with MI (Horne et al., 2007). This is congruent with our results. Recently, Nie et al. (2014) found that the MMP9 -1562 (rs3918242) $T$ allele was associated with a 1.5-fold increase in ischemic stroke risk in the Chinese population. Importantly, this finding is in line with our results.

The main strength of this study is the use of a coronary calcium score via computed tomography. This is a specific method to evaluate the coronary calcium level, and was, therefore, used to diagnose subclinical atherosclerosis. Indeed, the control individuals were selected if they had a coronary artery calcium $(C A C)$ level of zero $(C A C=0)$. We only selected patients where the Ml etiology presented was atherosclerotic plaque disruption. The reason for this was that the relevance of MMPs lies in the degradation of the extracellular matrix of the coronary plaque, including the thinning of the fibrous cap. This study strength must be considered when comparing our findings with similar studies.

However, some study limitations need to be addressed. Here, we only included the analysis of one polymorphism of each gene. Also, the findings were based on a limited sample size. We therefore suggest exercising caution when interpreting them.

In summary, our data suggest an association between a functional polymorphism in the promotor region of the MMP9 gene and the risk of developing MI in the Mexican Mestizo population. Although this is the largest study carried out in this population, our data are preliminary owing to the sample size. Consequently, additional studies in larger populations will be required to define the true genetic role of these polymorphisms in MI.

\section{Conflicts of interest}

The authors declare no conflict of interest.

\section{ACKNOWLEDGMENTS}

The authors and the Molecular Biology Department acknowledge the CONACyT for the support provided for this research (grants \#106012 and \#233402). The present project has been used as a Ph.D. degree for the corresponding author. The authors acknowledge the study participants and the personnel of the National Institute of Cardiology "Ignacio Chavez". Institutional Review Board approval was obtained for the sample collections and further analysis. 


\section{REFERENCES}

Alp E, Menevse S, Tulmac M, Kan D, et al. (2009). Lack of association between matrix metalloproteinase-9 and endothelial nitric oxide synthase gene polymorphisms and coronary artery disease in Turkish population. DNA Cell Biol. 28: 343-350. http://dx.doi.org/10.1089/dna.2009.0866

Alp E, Menevse S, Tulmac M, Yilmaz A, et al. (2011). The role of matrix metalloproteinase-2 promoter polymorphisms in coronary artery disease and myocardial infarction. Genet. Test. Mol. Biomarkers 15: 193-202. http://dx.doi.org/10.1089/ gtmb.2010.0113

Brown DL, Hibbs MS, Kearney M, Loushin C, et al. (1995). Identification of 92-kD gelatinase in human coronary atherosclerotic lesions. Association of active enzyme synthesis with unstable angina. Circulation 91: 2125-2131. http://dx.doi. org/10.1161/01.CIR.91.8.2125

Camacho-Mejorado R, Noris G, Santana C, Magaña JJ, et al. (2015). Interethnic variation of the MMP-9 microsatellite in Amerindian and Mexican Mestizo populations: considerations for genetic association studies. Genet. Mol. Res. 14: 29292939. http://dx.doi.org/10.4238/2015.March.31.24

Chaudhary AK, Pandya S, Ghosh K and Nadkarni A (2013). Matrix metalloproteinase and its drug targets therapy in solid and hematological malignancies: an overview. Mutat. Res. 753: 7-23.http://dx.doi.org/10.1016/j.mrrev.2013.01.002

Chehaibi K, Hrira MY, Nouira S, Maatouk F, et al. (2014). Matrix metalloproteinase-1 and matrix metalloproteinase-12 gene polymorphisms and the risk of ischemic stroke in a Tunisian population. J. Neurol. Sci. 342: 107-113. http://dx.doi. org/10.1016/j.jns.2014.04.036

Creemers EE, Cleutjens JP, Smits JF and Daemen MJ (2001). Matrix metalloproteinase inhibition after myocardial infarction: a new approach to prevent heart failure? Circ. Res. 89: 201-210. http://dx.doi.org/10.1161/hh1501.094396

Duellman T, Warren CL, Peissig P, Wynn M, et al. (2012). Matrix metalloproteinase-9 genotype as a potential genetic marker for abdominal aortic aneurysm. Circ Cardiovasc Genet 5: 529-537. http://dx.doi.org/10.1161/CIRCGENETICS.112.963082

Falk E (2006). Pathogenesis of atherosclerosis. J. Am. Coll. Cardiol. 47 (Suppl): C7-C12. http://dx.doi.org/10.1016/j. jacc.2005.09.068

Fang S, Jin X, Wang R, Li Y, et al. (2005). Polymorphisms in the MMP1 and MMP3 promoter and non-small cell lung carcinoma in North China. Carcinogenesis 26: 481-486. http://dx.doi.org/10.1093/carcin/bgh327

Herzog E, Gu A, Kohmoto T, Burkhoff D, et al. (1998). Early Activation of Metalloproteinases after Experimental Myocardial Infarction Occurs in Infarct and Non-infarct Zones. Cardiovasc. Pathol. 7: 307-312. http://dx.doi.org/10.1016/S10548807(98)00008-8

Horne BD, Camp NJ, Carlquist JF, Muhlestein JB, et al. (2007). Multiple-polymorphism associations of 7 matrix metalloproteinase and tissue inhibitor metalloproteinase genes with myocardial infarction and angiographic coronary artery disease. Am. Heart J. 154: 751-758.http://dx.doi.org/10.1016/j.ahj.2007.06.030

Lahiri DK and Nurnberger JI, Jr. (1991). A rapid non-enzymatic method for the preparation of HMW DNA from blood for RFLP studies. Nucleic Acids Res. 19: 5444. http://dx.doi.org/10.1093/nar/19.19.5444

Liu PY, Chen JH, Li YH, Wu HL, et al. (2003). Synergistic effect of stromelysin-1 (matrix metallo-proteinase-3) promoter 5A/6A polymorphism with smoking on the onset of young acute myocardial infarction. Thromb. Haemost. 90: 132-139.

Liu PY, Li YH, Tsai WC, Tsai LM, et al. (2005). Stromelysin-1 promoter 5A/6A polymorphism is an independent genetic prognostic risk factor and interacts with smoking cessation after index premature myocardial infarction. J. Thromb. Haemost. 3: 1998-2005. http://dx.doi.org/10.1111/j.1538-7836.2005.01515.x

Medley TL, Cole TJ, Dart AM, Gatzka CD, et al. (2004). Matrix metalloproteinase-9 genotype influences large artery stiffness through effects on aortic gene and protein expression. Arterioscler. Thromb. Vasc. Biol. 24: 1479-1484. http://dx.doi. org/10.1161/01.ATV.0000135656.49158.95

Neale BM and Sham PC (2004). The future of association studies: gene-based analysis and replication. Am. J. Hum. Genet. 75: 353-362. http://dx.doi.org/10.1086/423901

Newby AC (2005). Dual role of matrix metalloproteinases (matrixins) in intimal thickening and atherosclerotic plaque rupture. Physiol. Rev. 85: 1-31.http://dx.doi.org/10.1152/physrev.00048.2003

Nie SW, Wang XF and Tang ZC (2014). Correlations between MMP-2/MMP-9 promoter polymorphisms and ischemic stroke. Int. J. Clin. Exp. Med. 7: 400-404.

Nissi R, Talvensaari-Mattila A, Kotila V, Niinimäki M, et al. (2013). Circulating matrix metalloproteinase MMP-9 and MMP-2/ TIMP-2 complex are associated with spontaneous early pregnancy failure. Reprod. Biol. Endocrinol. 11: 2. http://dx.doi. org/10.1186/1477-7827-11-2

Olsen V, Rohde LE, Beck-da-Silva L, Santos KG, et al. (2014). QRS widening rates and genetic polymorphisms of matrix metalloproteinases in a cohort of patients with chronic heart failure. Can. J. Cardiol. 30: 345-351. http://dx.doi. org/10.1016/j.cjca.2013.11.014 
Opstad TB, Pettersen Å̊, Arnesen H and Seljeflot I (2013). The co-existence of the IL-18+183 A/G and MMP-9 -1562 C/T polymorphisms is associated with clinical events in coronary artery disease patients. PLoS One 8: e74498. http://dx.doi. org/10.1371/journal.pone.0074498

Opstad TB, Arnesen H, Pettersen Å̊ and Seljeflot I (2014). The MMP-9 -1562 C/T polymorphism in the presence of metabolic syndrome increases the risk of clinical events in patients with coronary artery disease. PLoS One 9: e106816. http:// dx.doi.org/10.1371/journal.pone.0106816

Pasterkamp G, Schoneveld AH, Hijnen DJ, de Kleijn DP, et al. (2000). Atherosclerotic arterial remodeling and the localization of macrophages and matrix metalloproteases 1, 2 and 9 in the human coronary artery. Atherosclerosis 150: 245-253. http:// dx.doi.org/10.1016/S0021-9150(99)00371-8

Qintao C, Yan L, Changhong D, Xiaoliang G, et al. (2014). Genetic polymorphism of matrix metalloproteinase-1 and coronary artery disease susceptibility: a case-control study in a Han Chinese population. Genet. Test. Mol. Biomarkers 18: 826831. http://dx.doi.org/10.1089/gtmb.2014.0222

Ratnikov BI, Cieplak P, Gramatikoff K, Pierce J, et al. (2014). Basis for substrate recognition and distinction by matrix metalloproteinases. Proc. Natl. Acad. Sci. USA 111: E4148-E4155.http://dx.doi.org/10.1073/pnas.1406134111

Román-García P, Coto E, Reguero JR, Cannata-Andía JB, et al. (2009). Matrix metalloproteinase 1 promoter polymorphisms and risk of myocardial infarction: a case-control study in a Spanish population. Coron. Artery Dis. 20: 383-386. http:// dx.doi.org/10.1097/MCA.0b013e32832fa9cf

Sakowicz A, Fendler W, Lelonek M, Sakowicz B, et al. (2013). Genetic polymorphisms and the risk of myocardial infarction in patients under 45 years of age. Biochem. Genet. 51: 230-242. http://dx.doi.org/10.1007/s10528-012-9558-5

Sewelam NI, Radwan ER, Andraos AW, Ibrahim BE, et al. (2013). Association between the polymorphisms of matrix metalloproteinases 9 and 3 genes and risk of myocardial infarction in Egyptian patients. Egypt. J. Med. Hum. Genet. 14: 143-148. http://dx.doi.org/10.1016/j.ejmhg.2013.01.005

Wang S, Dai YX, Chen LL, Jiang T, et al. (2015). Effect of IL-1 $\beta$, IL-8, and IL-10 polymorphisms on the development of myocardial infarction. Genet. Mol. Res. 14: 12016-12021. http://dx.doi.org/10.4238/2015.October.5.14

Yang J, Lin S, Zhou J, Wu B, et al.; Chengdu Stroke Registry and Nanjing First Hospital Stroke Registry investigators (2014). Genetic variations of MMP9 gene and intracerebral hemorrhage outcome: a cohort study in Chinese Han population. $J$. Neurol. Sci. 343: 56-59.http://dx.doi.org/10.1016/j.jns.2014.05.021

Ye S (2000). Polymorphism in matrix metalloproteinase gene promoters: implication in regulation of gene expression and susceptibility of various diseases. Matrix Biol. 19: 623-629. http://dx.doi.org/10.1016/S0945-053X(00)00102-5

Zhi H, Wang H, Ren L, Shi Z, et al. (2010). Functional polymorphisms of matrix metallopeptidase-9 and risk of coronary artery disease in a Chinese population. Mol. Biol. Rep. 37: 13-20. http://dx.doi.org/10.1007/s11033-009-9482-x 